\title{
Hollow Cathode Thruster for All-Electric Spacecraft
}

\author{
Paolo Gessini ${ }^{1}$, Michele Coletti ${ }^{2}$, Angelo Grubisic ${ }^{3}$ and Stephen B. Gabriel ${ }^{4}$ \\ University of Southampton, Southampton, Hants, SO17 1BJ, United Kingdom \\ Neil C. Wallace ${ }^{5}$ \\ Space Department, QinetiQ, Farnborough, Hants, GU14 OLX, United Kingdom \\ and \\ David G. Fearn ${ }^{6}$ \\ EP Solutions, Fleet, Hants, GU52 6HS, United Kingdom
}

\begin{abstract}
The thrust produced by a T6 ion engine hollow cathode was characterized using different propellants with a target-based measurement system developed at the University of Southampton, for discharge current values of 5-25 A and a wide range of mass flow rates. The calculated values of specific impulse are far in excess of those that could be attributed to heating a gas to thermal equilibrium with the walls, which seems to suggest an arcjet-like operation mechanism. A 0-th order model was developed, which already seems to capture several essential features, and the discharge power per unit mass flow rate (specific power) appears to be the mean scaling parameter for the specific impulse. A hollow cathode thruster could be used, in conjunction with an ion engine, to propel an all-electric spacecraft.
\end{abstract}

\section{Nomenclature}

$=$ ion sound speed

$=$ beam width

$=$ hollow cathode orifice diameter

$=$ target diameter

$=$ electron charge

$=$ Young's modulus

$=$ thrust

$=$ acceleration of gravity at sea level

$=$ beam thickness

$=$ discharge current

$=$ specific impulse

$=$ Boltzmann constant

$=$ hollow cathode orifice length

$=$ beam length

$=$ atomic mass

$=$ mass flow rate

$=$ ion Mach number

$=$ number density

$=$ optical path length

$=$ plasma resistance

\footnotetext{
${ }^{1}$ Visiting Researcher, Astronautics Research Group, Southampton, Hants SO17 1BJ, UK, AIAA Member.

${ }^{2} \mathrm{PhD}$ Student, Astronautics Research Group, Southampton, Hants SO17 1BJ, UK, AIAA Member.

${ }^{3} \mathrm{PhD}$ Student, Astronautics Research Group, Southampton, Hants SO17 1BJ, UK, AIAA Member.

${ }^{4}$ Professor, Astronautics Research Group, Southampton, Hants SO17 1BJ, UK, AIAA Member.

${ }^{5}$ Electric Propulsion Manager, Space Department, Farnborough, Hants, GU14 0LX, UK, AIAA Member.

${ }^{6}$ Sole Proprietor, 23 Bowenhurst Road, Church Crookham, Fleet, Hants GU52 6HS, UK, AIAA Senior Member.
} 


$\begin{array}{ll}T & =\text { heavy-particle temperature } \\ T_{e} & =\text { electron temperature } \\ v_{i} & =\text { ion velocity } \\ V & =\text { discharge voltage } \\ V_{P} & =\text { plasma voltage } \\ V_{S} & =\text { sheath voltage } \\ \gamma & =\text { specific heat ratio } \\ \Delta \theta & =\text { angular deflection } \\ \Delta x & =\text { linear displacement } \\ \eta & =\text { plasma resistivity }\end{array}$

\section{Introduction}

$\mathrm{H}^{\circ}$ OLLOW Cathodes (HCs) have been used for decades in various electric propulsion applications and have been extensively investigated and tested, demonstrating excellent performance and durability, with tens of thousands of hours of successful operation. Experimental programs over the past two or three decades have aided greatly in the understanding and modeling of the HC discharge physical mechanisms, particularly when using xenon ${ }^{1,2}$. The thrust generated by a $\mathrm{HC}$ in isolation, however, had only been actually measured in one instance, with a device, operating on mercury, quite different from the present-day $\mathrm{HCs}^{3}$, until recently the thrust from a QinetiQ T6 ion engine $\mathrm{HC}$ was characterized at the University of Southampton ${ }^{2,4}$. The HC was a standard T6 cathode and therefore the design was not optimized for operation as a HC Thruster (HCT), the test program being intended solely to examine the feasibility of employing a $\mathrm{HC}$ as a thruster.

\section{Experimental Apparatus}

Experiments were carried out in the Astronautics Laboratory at the University of Southampton, School of Engineering Sciences, Astronautics Research Group, in order to evaluate for what discharge conditions the highest values of specific impulse and in general the best performance were attained.

\section{A. Measurement System}

The thrust measurement system developed in-house at the University of Southampton consists of a Cantilever Beam Target (CBT), a Laser Optical Lever (LOL) and a Position Sensitive Detector (PSD). A photograph of the CBT and a schematic of the LOL are shown in Fig. 1.

The HC plume impinges on the CBT, mounted downstream of the anode in a horizontal position, as shown in Fig. 2, in order to decouple the effects of $\mathrm{HC}$ thrust and target weight. The target angular deflection $\Delta \theta$ is related to the thrust $F$ by $^{2,5}$

$$
\frac{\Delta \theta}{F}=\frac{6}{E b h^{3}} L(L+D)
$$

where $E$ is the Young's modulus of the material used (molybdenum for this CBT) and the other symbols are illustrated in Fig.1. $\Delta \theta$ is translated into a linear displacement $\Delta x$ with a Laser Optical Lever (LOL), according to the formula ${ }^{2,5}$

$$
\Delta x=2 P \Delta \theta,
$$

valid for small values of $\Delta \theta$ (exaggerated in Fig. 1), where $P$ is the optical path length. $F$ is therefore related to $\Delta x$ by the expression ${ }^{2,5}$

$$
F=\frac{E b h^{3}}{12 P L(L+D)} \Delta x
$$


$\Delta x$ is measured with a Hamamatsu S2044 Position Sensitive Detector (PSD), with a resolution of $600 \mathrm{~nm}$, corresponding, in the present configuration (with an optical path length $P \sim 1 \mathrm{~m}$ ), to a thrust measurement resolution of $\sim 3 \mu \mathrm{N}^{2,5}$.

\section{B. Vacuum Rig}

The vacuum rig used in the experiments reported in this paper was described in previous works, and has undergone only minor modifications since then ${ }^{2}$. It consists of a stainless steel cylindrical chamber, $0.5 \mathrm{~m}$ in diameter and 0.5 $\mathrm{m}$ long, pumped down to a pressure in the $10^{-7}$ mbar range (with no flow) by the use of a rotary and a turbomolecular pump connected in series.

\section{Hollow Cathode and Anode Assembly}

The HC investigated was developed by QinetiQ for the T6 Kaufman-type ion thruster. Its basic features have essentially remained unchanged since the start of the program and are described, together with the physics of $\mathrm{HC}$ operation, in various literature ${ }^{2,7,8,9}$. The original orifice, $0.5 \mathrm{~mm}$ in diameter, was later drilled out to a $1.0 \mathrm{~mm}$ diameter. The $\mathrm{HC}$ was operated in an open-diode configuration, without using a separate keeper electrode as in the majority of previous investigations in the literature. A circular anode, machined from a $100 \mathrm{~mm}$ diameter steel disc, is mounted on insulating supports at $\sim 2 \mathrm{~mm}$ in front of the $\mathrm{HC}$ tip, as shown in Fig. 2. The anode presents a large $(20-40 \mathrm{~mm}$ diameter) central orifice, to allow the plume to reach the target relatively unimpeded.
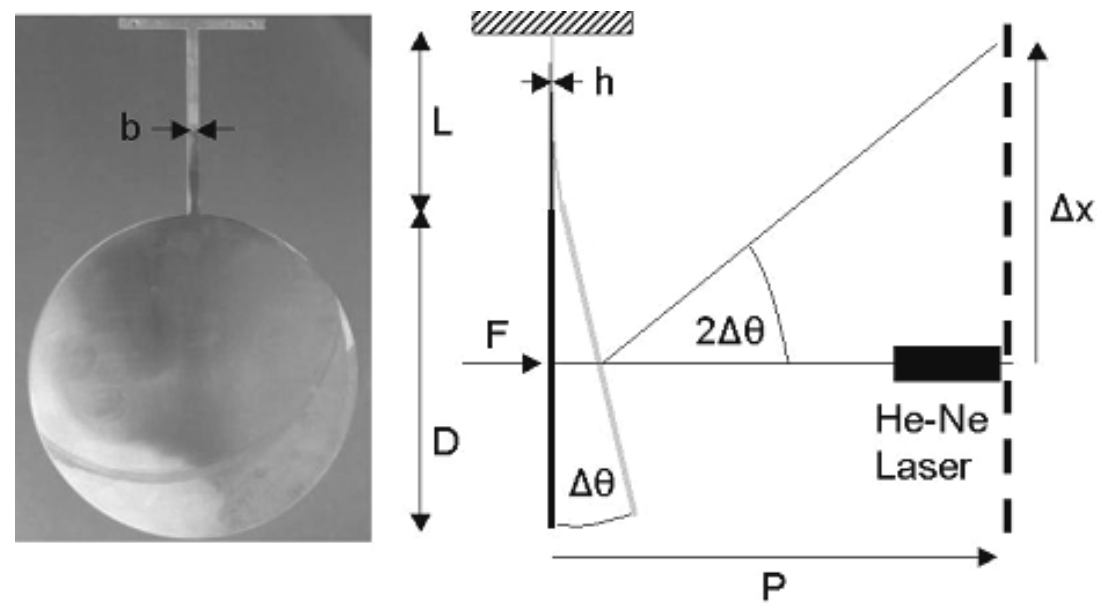

Figure 1. Photo of the CBT and schematic of the LOL.

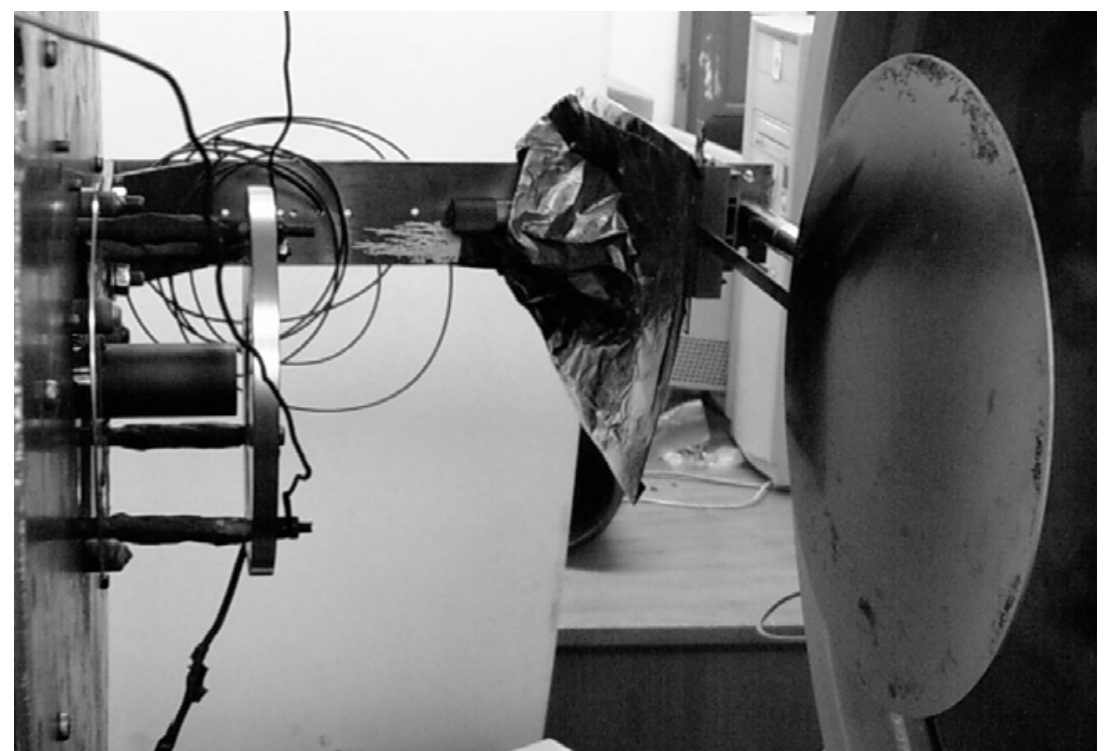

Figure 2. Photo of the T6 HC in open-diode configuration, with the CBT mounted in front of it, on the right.

\section{Results and Discussion}

The HC was operated in open-diode configuration, with a single anode at $\sim 2 \mathrm{~mm}$ in front of the HC tip. The anode presented a large orifice, in order for the plume to reach the target relatively unimpeded. Extensive thrust measurements, taken with different cathode $(0.5 \mathrm{~mm}$ and $1.0 \mathrm{~mm})$ and anode $(20 \mathrm{~mm}$ and $40 \mathrm{~mm})$ orifice diameters and various noble gases ( $\mathrm{Ar}, \mathrm{Kr}$ and $\mathrm{Xe}$ ) as propellants, were presented and discussed in Ref. 4.

\section{General Considerations}

Our analysis of the results obtained suggests that the $\mathrm{HC}$ in open-diode configuration generates thrust mainly through electrothermal acceleration mechanisms. The specific impulse values obtained confirm that the device is not just heating the gas, like a resistojet, but is operating in a fashion more similar to that of an arcjet, with the heavy 
particle average temperature sensibly higher than the wall value. Even assuming full conversion of the flow enthalpy into directed kinetic energy, neglecting the plume divergence and other losses, we would in fact obtain

$$
I_{s p}=\frac{F}{\dot{m g}}=\frac{1}{g} \sqrt{\frac{5 k T}{m}},
$$

where $\dot{m}$ is the mass flow rate, $g$ the acceleration of gravity at sea level, $k$ the Boltzmann constant, $T$ the heavyparticle temperature and $m$ the atomic mass. Eq. (4) yields, for argon at $2000 \mathrm{~K}$ (a temperature probably higher than that of the insert wall), a value of $I_{s p} \sim 150 \mathrm{~s}$, which is an upper limit, unattainable in practice because of various kinds of losses. As the measured thrust yields values of specific impulse that are much higher (up to $\sim 500 \mathrm{~s}$ ) for certain discharge current and mass flow rate conditions, as shown in Fig. 3, we conclude that energy is transferred to the flow through different mechanisms. In particular, intense heating of the plasma, mainly by ohmic dissipation, probably takes place in the orifice, where the heavy-particle temperature in the flow core is raised to values well above the wall temperature and electron temperatures can be as high as a few $\mathrm{eV}$, as recently measured at $\mathrm{JPL}^{10}$.

As the pressure in the vacuum chamber stayed extremely low even during $\mathrm{HC}$ operation at the highest mass flow rates, we can assume that the orifice flow remained constantly choked, as also confirmed by numerical simulations ${ }^{11}$. This would of course be all the more the case during actual operation of a HCT in space. Heat addition in the insert and especially in the orifice regions does not change the situation, as it

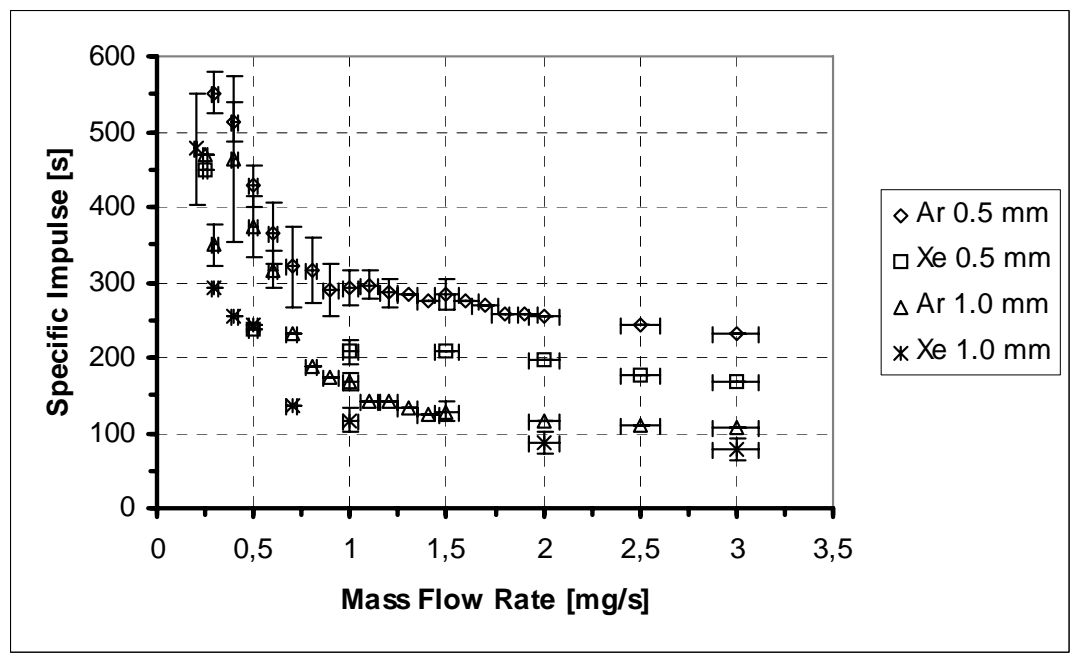

Figure 3. Isp dependence on mass flow rate at $25 \mathrm{~A}$ discharge current with different propellants and $\mathrm{HC}$ orifice diameters.

also tends to choke a subsonic flow (Rayleigh flow). The fact that we are dealing here with a plasma, and not a neutral fluid, yields the following expression for the ion Mach number ${ }^{12}$ :

$$
M_{i}=\frac{v_{i}}{a_{i}}=\frac{v_{i}}{\sqrt{\frac{\gamma k T_{e}}{m}}}=\frac{v_{i}}{\sqrt{\frac{5 k T_{e}}{3 m}}},
$$

where $v_{i}$ is the ion velocity, $a_{i}$ the ion sound speed and Te the electron temperature. In Eq. (5) we assume that the ions are singly ionized and neglect the difference between atomic and ion mass. We also use the value of the specific heat ratio $\gamma=5 / 3$ for a monatomic gas. If, then, the ionization fraction is high, a $M=1$ condition at the exit of the orifice will correspond to a heavy-particle velocity essentially equal to $a_{i}$. It is therefore instructive to see what electron temperatures in the orifice would correspond to sonic speeds compatible with the values of specific impulse that were actually measured. The data shown in Fig. 3 correspond to values of $T e$ of a few eV in spot mode ${ }^{13}$, rising quickly to several $\mathrm{eV}$ as the $\mathrm{HC}$ moves into plume mode ${ }^{13}$ operation, in fair agreement with JPL measurements ${ }^{10}$. This suggests that the flow is indeed highly ionized, especially for smaller orifice diameters, corresponding to higher current densities, with an average heavy-particle exhaust velocity much higher than the orifice wall temperature.

\section{E. $0^{\text {th }}$-Order Modeling}

As the $\mathrm{HC}$ orifice dimensions are small, but still much bigger than the ion and electron mean free paths, this can be modeled, as a fist approximation, as a uniform plasma cylinder, thus neglecting sheath phenomena. By assuming full ionization inside the orifice and using a classical, Spitzer value for the plasma resistivity ${ }^{12}$, we can calculate the power dissipated in the orifice plasma. A first constraint is that this amount of power has to be lower than the total 
discharge power, as a sizable part of the voltage fall happens in the sheaths at the $\mathrm{HC}$ walls. As the resistivity, under our assumptions, depends only on the electron temperature $T e$, being proportional to $T e^{-3 / 2}$, this constraint means that $T e$ has to be much higher than $1 \mathrm{eV}$ (the value generally accepted for the insert plasma), in general, to yield low enough values for the resistivity. Part of this dissipated power then energizes the heavy particles and is converted into thrust. As stated above, the measured values of $I_{s p}$ imply that the average value of $T$ has to be much higher than the wall temperature. If we assume, as a first approximation, the efficiency of the conversion of dissipated power into thrust to be a constant, at least for a given propellant gas, we can write

$$
R_{P} I^{2}=\eta \frac{l}{\pi\left(\frac{d}{2}\right)^{2}} I^{2} \propto \frac{1}{2} \dot{m} v_{e}^{2}=\frac{1}{2} \dot{m}\left(g I_{s p}\right)^{2},
$$

where $R_{P}$ is the resistance of the plasma cylinder, $v_{e}$ the exhaust velocity, $\eta$ the plasma resistivity, $l$ the $\mathrm{HC}$ orifice length, $d$ the $\mathrm{HC}$ orifice diameter and $I$ the discharge current. Eq. (6) yields:

$$
\eta I\left(\frac{I}{d}\right)^{2} \propto \dot{m} I_{s p}^{2}
$$

and, after rearranging:

$$
I_{s p} \propto \frac{I}{d} \sqrt{\frac{\eta l}{\dot{m}}} .
$$

In our experiments the length of the $\mathrm{HC}$ orifice $l$ did not vary more than $25 \%$. As it appears in Eq. (8) under square root, we will neglect the dependence on $l$. If now, for ease of analysis, we further assume the resistivity to vary little, so that its square root can be considered to be constant, Eq. (8) reduces to

$$
I_{s p} \propto \frac{I}{d \sqrt{\dot{m}}}
$$

This is not unreasonable for a fully ionized flow, following a Spitzer-type law for resistivity ${ }^{12}$, as $\eta$ will vary as $T e^{-3 / 2}$, so a doubling of $T_{e}$, for example, will give only a factor of three reduction in resistivity and a $40 \%$ decrease in $\sqrt{\eta}$. Equation (9) implies that, at constant current, for the same orifice diameter the specific impulse is inversely proportional to the square root of the mass flow rate while, for the same mass flow rate, the specific impulse is inversely proportional to the orifice diameter. As shown in Fig. 3, both these trends can be observed in our experimental results, at least for high mass flow rates, when the $\mathrm{HC}$ discharge is in spot mode. For the same orifice diameter and mass flow rate, on the other hand, Eq. (9) predicts a direct proportionality between discharge current and specific impulse. This trend is also confirmed by our experimental results. Fig. 4 shows the proportionality between $I_{s p}$ and $I$ to be verified quite accurately at higher mass flow rates and discharge currents, where the discharge is in spot mode or quiet plume mode, while for noisy, unstable plume mode a more irregular behavior can be observed. At low values of mass flow rate (plume mode operation) the specific impulse increases greatly and the differences in $\mathrm{HC}$ orifice diameter become less important. Interestingly, our $0^{\text {th }}$-order modeling already captures some essential features of HCT operation. 
It is instructive to plot the specific impulse vs. the RHS of Eq. (9), as shown in Figs. 5 and 6 for argon and xenon, respectively. The regions where the behavior is not approximately linear will correspond to the operating conditions where our assumptions are not valid. The percentage of dissipated power that will go to increase the heavy-particle temperature, and the efficiency of the conversion of this into directed kinetic energy, in fact, will vary with different discharge conditions. Furthermore, $\eta$ itself may not follow a Spitzer-type law, because anomalous resistivity is important or the plasma is not fully ionized, and therefore it may be a function of $T_{e}$ and of other plasma parameters. Thus our assumption of nearconstant plasma resistivity will no longer be valid, and the ohmic dissipation may vary considerably. We notice a considerable spread in the results, which is considerably reduced when only the measurements taken in spot mode are plotted, as in Figs. 7 and 8. This confirms that our simple analysis is applicable to spot mode operation and maybe quiet, lower-voltage plume mode, but is inadequate to explain high-voltage plume mode phenomena, where the discharge becomes noisy and the operation unstable.

From Fig. 3, in fact, it is apparent that, as the mass flow rate decreases, and the discharge moves into plume mode, with rapidly increasing voltages, the $I_{s p}$ increases sharply. As we are operating at constant current, increasing voltages mean increasing discharge power. The specific power, i.e., the power per unit mass flow rate, thus also increases rapidly.

In spot mode operation, the voltage drop across the plasma outside the $\mathrm{HC}$ and the anode fall are small compared to the voltage drop across the ideal plasma cylinder considered in our model, $V_{P}$, and the voltage drop across the

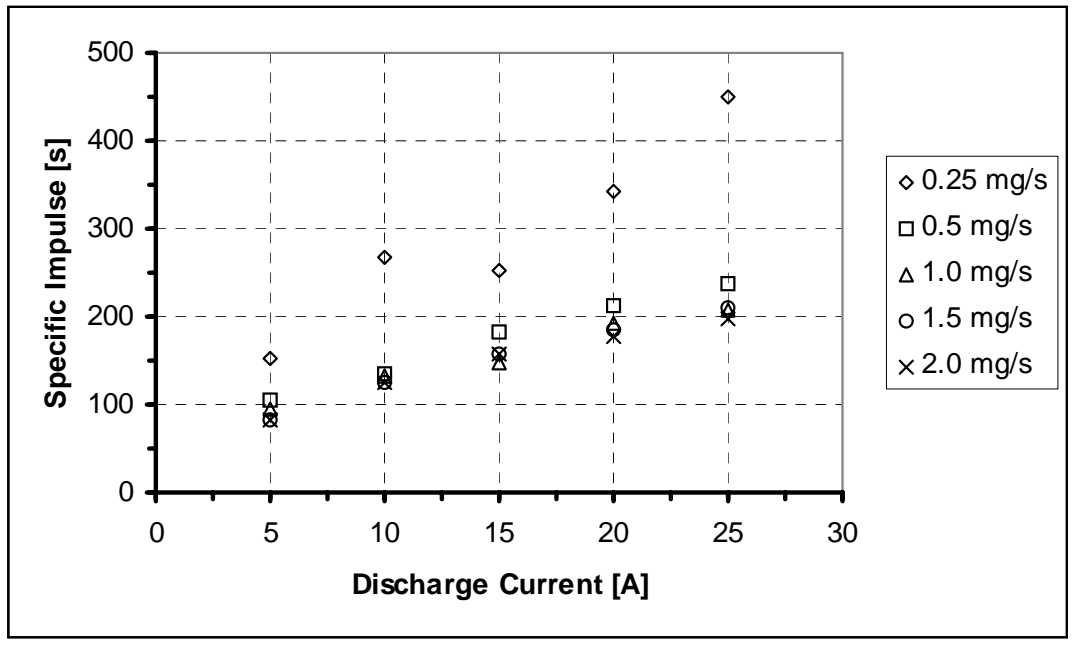

Figure 4. Isp dependence on discharge current at constant values of mass flow rate, xenon, $0.5 \mathrm{~mm}$ HC orifice diameter.

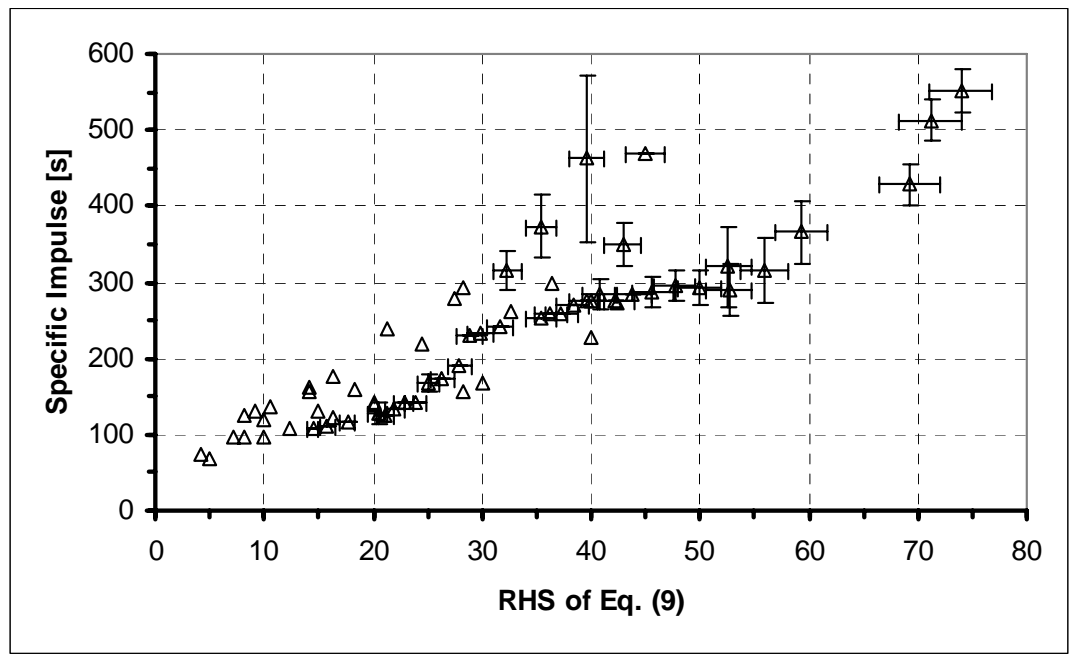

Figure 5. Isp plotted vs. the RHS of Eq. (9), argon.

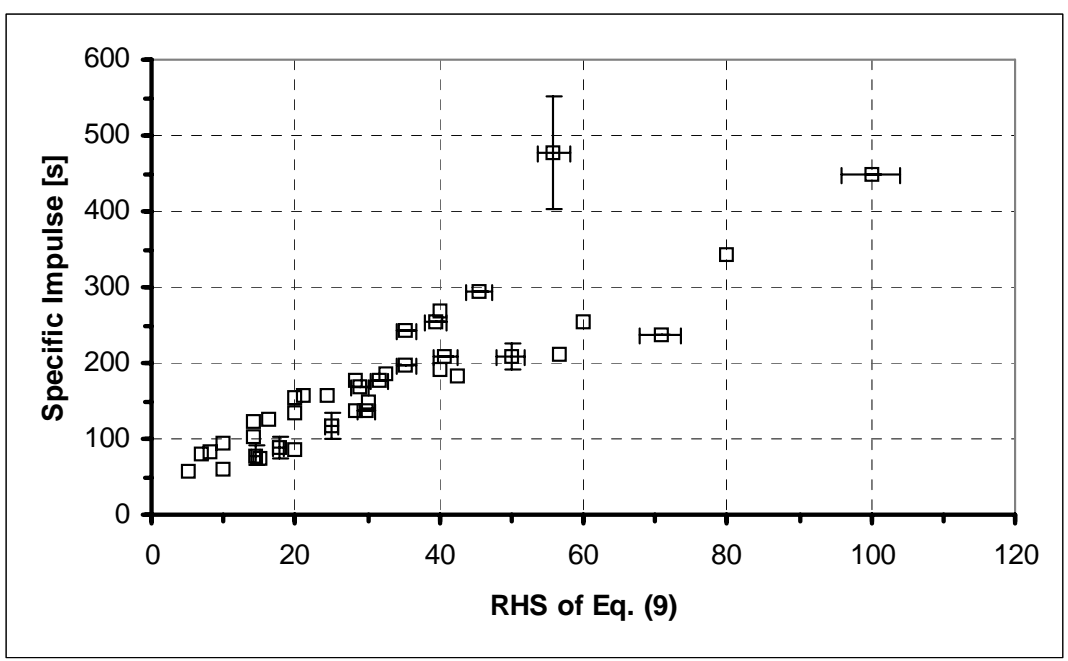

Figure 6. Isp plotted vs. the RHS of Eq. (9), xenon. 
$\mathrm{HC}$ wall sheath, $V_{S}$. Applying Ohm's law and expressing the plasma cylinder resistance as in Eq. (6), we can then write the total discharge voltage $V$ as

$$
V=V_{P}+V_{S}=R_{P} I+V_{S}=\eta \frac{l}{\pi\left(\frac{d}{2}\right)^{2}} I+V_{S} .
$$

The discharge power can thus be written as

$$
V I=\left(V_{P}+V_{S}\right) I=R_{P} I^{2}+V_{S} I=\eta \frac{l}{\pi\left(\frac{d}{2}\right)^{2}} I^{2}+V_{S} I
$$

From Eqs. (10) and (11) we see that in spot mode, with the discharge voltage $V$ sensibly constant and an $\eta$ that varies little, the power dissipated in the plasma cylinder will approach the total discharge power with increasing current, as the sheath voltage drop $V_{S}$ will decrease. This can be physically explained as follows.

The current is exponentially related to the potential at the $\mathrm{HC}$ wall (Schottky effect) ${ }^{12}$, so only a very small increase in sheath voltage would be required to go from $5 \mathrm{~A}$ to $25 \mathrm{~A}$, under constant plasma conditions. An increase in current leads to an increase in the power dissipation in the sheath, so the cathode temperature rises (as we see experimentally), emission is thereby enhanced, and the $\mathrm{HC}$ sheath voltage falls (in the absence of changes in plasma parameters). The sheath thickness is related to the Debye length, which is proportional to the square root of the electron temperature divided by the square root of the number density $n_{e}{ }^{12}$. The electron temperature will increase only slowly with the current, but the number density will increase rapidly with current, due to the larger number of carriers required, and with flow rate. So the sheath will become thinner as current and flow increase, enhancing the emission current and offsetting the need for a higher sheath voltage. So the sheath voltage also falls for this reason. The plasma conditions are probably very different in plume mode, with a lower $n_{e}$ and higher $T_{e}$ than in the spot mode. So the sheath will be thicker, requiring a higher applied

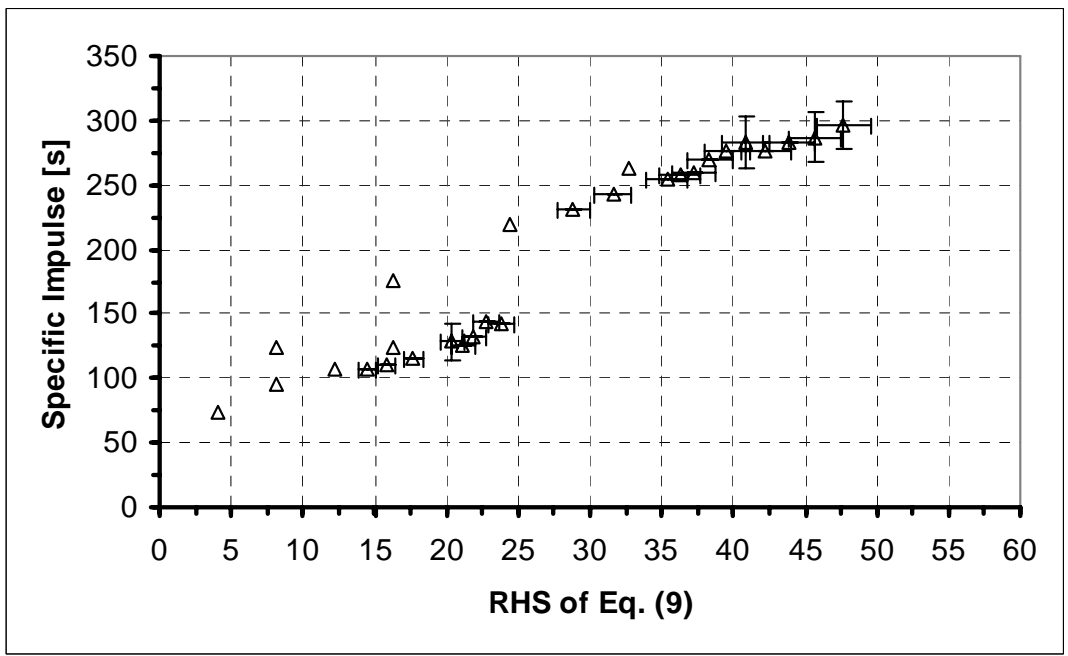

Figure 7. Isp plotted vs. the RHS of Eq. (9), argon, spot mode.

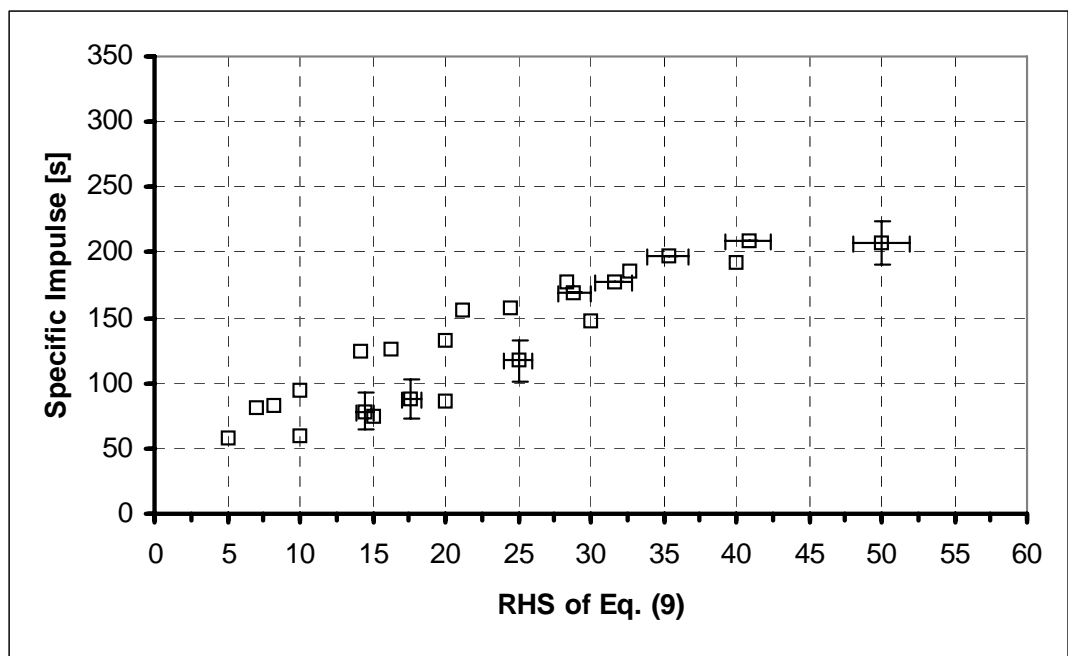

Figure 8. Isp plotted vs. the RHS of Eq. (9) xenon, spot mode. 
voltage for a given current, as found in practice.

Equation (6) can then be rewritten, for high values of discharge current, as

$$
V I \propto \frac{1}{2} \dot{m} v_{e}^{2}=\frac{1}{2} \dot{m}\left(g I_{s p}\right)^{2},
$$

which yields

$$
I_{s p} \propto \sqrt{\frac{V I}{\dot{m}}} .
$$

Equation (13) predicts that, at high currents, the specific impulse should be proportional to the square root of the specific power, at least for a HCT operating in spot mode, and the dependence on $\mathrm{HC}$ orifice diameter should tend to disappear. In Figs. 9 and 10 we can see that the correlation between $I_{s p}$ and the square root of the specific power is indeed becoming rather linear, both with argon and xenon as propellants, at high values of discharge current (to avoid crowding the plots, only the measurements at $25 \mathrm{~A}$ discharge current are shown) and specific power, with little difference between 0.5 and $1.0 \mathrm{~mm} \mathrm{HC}$ orifice diameter. As before, a noticeable spread in the data can be observed at the highest values of specific power, corresponding to high-voltage, noisy plume-mode operation at very low mass flow rates. This trend may be used to improve the HCT performance, if high values of specific power can be obtained with modest levels of power at low mass flow rates.

\section{Conclusion}

These encouraging results were obtained just by varying the discharge parameters, with no attempt at optimizing the electrode configuration beyond the $\mathrm{HC}$ and

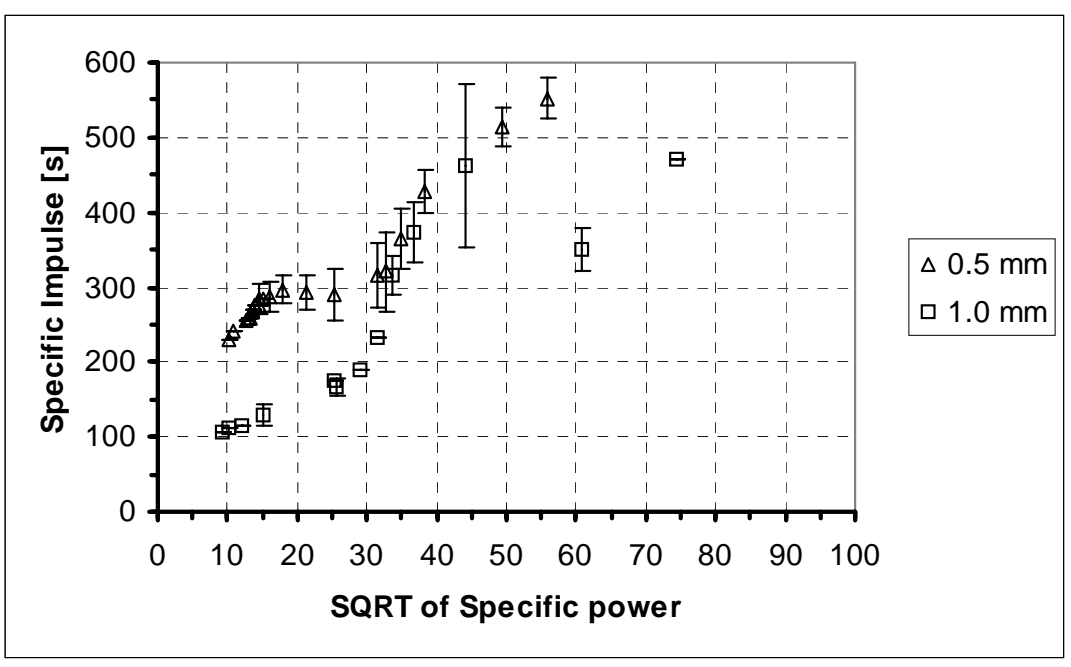

Figure 9. Isp plotted vs. the square root of specific power, argon, $25 \mathrm{~A}$.

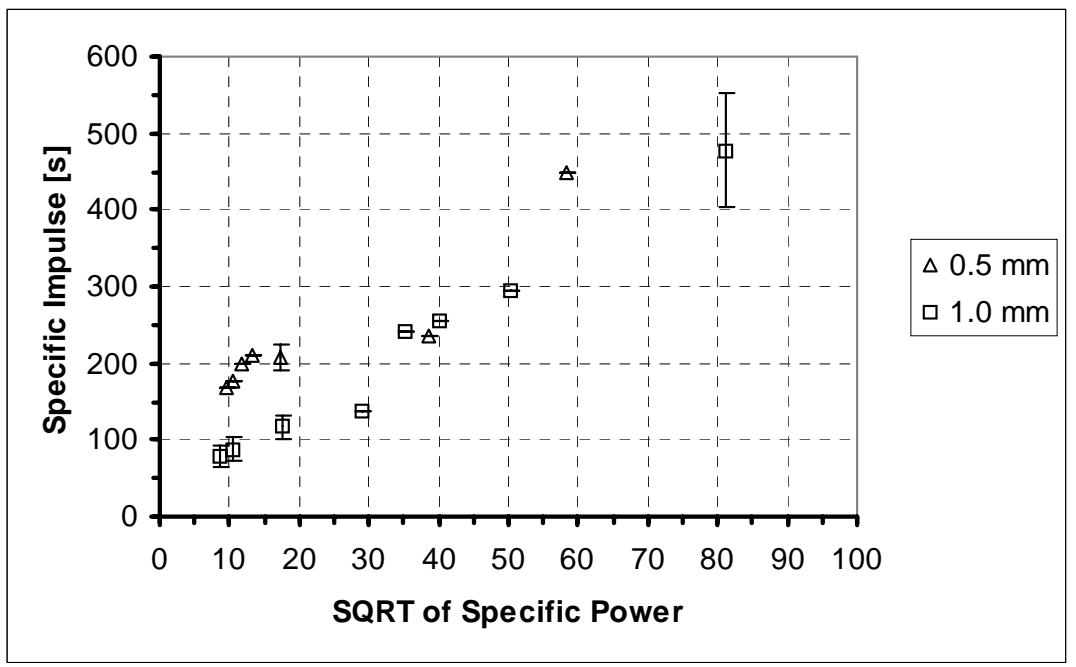

Figure 10. Isp plotted vs. the square root of specific power, xenon, $25 \mathrm{~A}$. anode orifice variation. Whereas, as shown in Fig. 3, a smaller orifice diameter and the use of lower-atomic weight propellants proved beneficial, from Figs. 9 and 10 the specific power appears to be the main scaling parameter for $I_{s p}$. The HCT performance could be further improved by increasing the specific impulse and the thrust-to-power ratio. The T6 HC is closely derived from the UK-25 model, which was tested with up to $50 \mathrm{~A}$ discharge currents ${ }^{14}$. It is thus to be expected that operation will be possible at current values considerably higher than $25 \mathrm{~A}$. The 
investigation of different electrode configurations and of other propellants could lead to the development of a viable HC microthruster, with $I_{s p}$ values competitive with already existing devices in the same input power range. In particular, the use of an enclosed keeper and of a cylindrical/conical anode to maintain low-voltage, stable spot mode operation even at low mass flow rates, is being investigated at the University of Southampton. With such a discharge configuration it is possible to achieve at a lower power consumption the values of specific power, and therefore specific impulse, only found during plume mode operation in the experiments described in this paper. A possible preliminary design for a $\mathrm{HCT}$ is shown in Fig. 11, from Ref. 15.

Although the possibility exists of increasing attainable values of specific impulse, it is unlikely that significant improvements will be made in thrust efficiency due to convective and radiative losses in the system. HCTs are therefore unlikely to compete with high $I_{s p}$, high thrust efficiency systems such as gridded ion engines and hall thrusters, however they will be able to operate at low power and with relatively low cost and complexity. The extensive space heritage and qualification status of a large number of HCs also provides a good knowledge base for
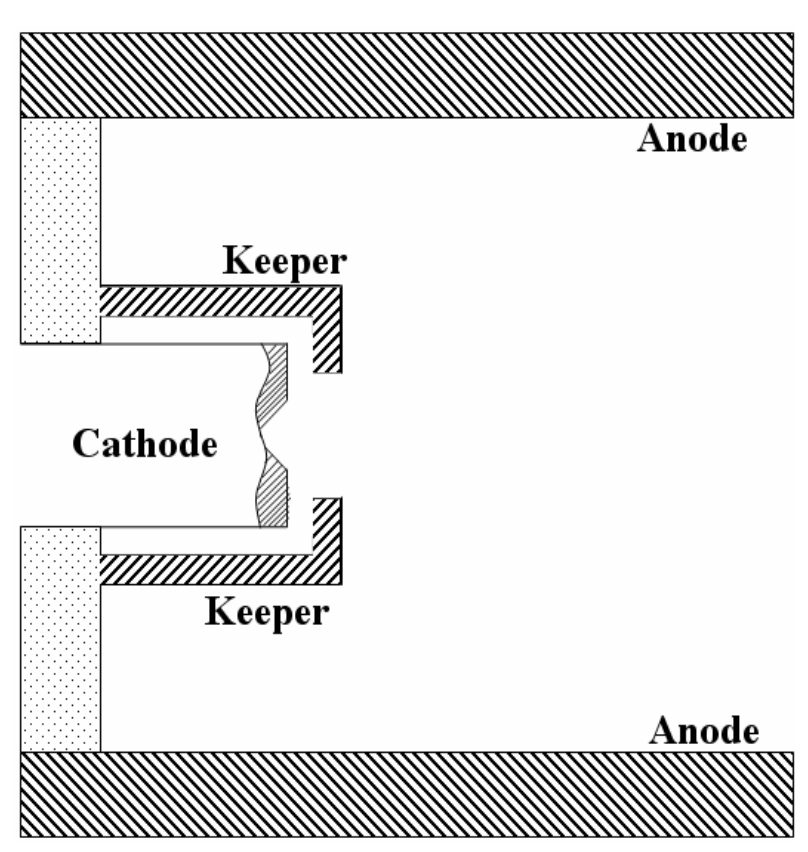

Figure 11. Preliminary design of a HCT, with enclosed keeper and cylindrical anode ${ }^{15}$. the development of long life thrusters based on previous HC designs. Investigation into the thrust production processes are being continued at the University of Southampton with the aim of optimizing design ${ }^{16}$. This development is based on the T6 (operating up to $800 \mathrm{~W}$ ) and T5 (operating up to $100 \mathrm{~W}$ ) HCs for both high and low power operation.

In a small-to-medium size spacecraft, the use of HCTs for attitude control could be convenient, even at modest values of $I_{s p}$, in conjunction with an ion engine for primary propulsion, as these two devices could share the same propellant (most likely xenon), flow control systems and power conditioning subsystems. This would avoid the need for a separate chemical propulsion system. Such an "all-electric spacecraft" would be considerably lighter and simpler, due to a reduced number of subsystems and to propellant mass savings made possible through the higher $I_{s p}$. It could present different levels of integration between the main propulsion system and the HCTs, depending on the possibility of using ad-hoc designed hardware. The higher the integration, the lower will be the mass of the satellite, but the higher will be the cost of the mission, hence a trade-off must be done. A relatively cheap and light solution consists in using a shared tank and flow control unit but keeping two distinct power processing unit. A mission that is taking into account the possibility of using this kind of thruster is the European Student Moon Orbiter (ESMO) mission by SSETI/ESA. A paper presenting a complete electric propulsion subsystem design study is in preparation $^{17}$.

\section{References}

${ }^{1}$ Gessini, P., Gabriel, S. B., and Fearn, D. G., "The Hollow Cathode as a Micro-Ion Thruster," $27^{\text {th }}$ International Electric Propulsion Conference, IEPC Paper 01-233, 2001 and References therein.

${ }^{2}$ Gessini, P., "Measurement of the Thrust Generated by a Hollow Cathode with Noble Gases," Ph.D. Dissertation, Aerospace Engineering Department, University of Southampton, Southampton, UK, 2006.

${ }^{3}$ Snyder, A., and Banks, B. A., "Thrust Measurements of a Hollow-Cathode Discharge," NASA TN D-6705, 1972.

${ }^{4}$ Gessini, P., Gabriel, S. B., and Fearn, D. G., "A Study of the Thrust Generated by a T6 Hollow Cathode," AIAA/ASME/SAE/ASEE 42 ${ }^{\text {nd }}$ Joint Propulsion Conference \& Exhibit, AIAA Paper 2006-5265, 2006 and References therein.

${ }^{5}$ Gessini, P., Gabriel, S. B., and Fearn, D. G., "Hollow Cathode Thrust Measurements Using a Target," $4^{\text {th }}$ International Spacecraft Propulsion Conference, ISPC Paper 04-230, 2004 and References therein.

${ }^{6}$ Gessini, P., Gabriel, S. B., and Fearn, D. G., "Hollow Cathode Thrust Measurement Using a Target: System Development and Calibration," AIAA/ASME/SAE/ASEE 38 th Joint Propulsion Conference \& Exhibit, AIAA Paper 2002-4104, 2002 and References therein.

${ }^{7}$ Gessini, P., Gabriel, S. B., and Fearn, D. G., "Hollow Cathode Thrust Measurements Using a Target: Initial Results and Some Issues," $28^{\text {th }}$ International Electric Propulsion Conference, IEPC Paper 03-253, 2003 and References therein. 
${ }^{8}$ Patterson, S. W., and Fearn, D. G., "The Generation of High Energy Ions in Hollow Cathode Discharges," $26^{\text {th }}$ International Electric Propulsion Conference, IEPC Paper 99-125, 1999 and References therein.

${ }^{9}$ Fearn, D. G., and Patterson, S. W., "Characterisation of the High Current Hollow Cathode for the T6 Ion Thruster," AIAA/ASME/SAE/ASEE 34th Joint Propulsion Conference \& Exhibit, AIAA Paper 98-3346, 1998.

${ }^{10}$ Goebel, D. M., Jameson, K. K., Watkins, R. M., Katz, I., andMikellides, I. G., "Hollow Cathode Theory and Experiment. I. Plasma Characterization Using Fast Miniature Scanning Probes," J. Appl. Phys., Vol. 98, 113302, 2005, pp. 1-9.

${ }^{11}$ Crawford, F. T. A., "A Numerical Model of the Ion Thruster Hollow Cathode Plasma," Ph.D. Dissertation, Aerospace Engineering Department, University of Southampton, Southampton, UK, 2004.

${ }^{12}$ Huba, J. D., "NRL Plasma Formulary," Revised, Naval Research laboratory, Washington, DC, 2000.

${ }^{13}$ Rudwan, I. M. A., "Physics of Hollow Cathode Breakdown and Steady-State Operation with Several Inert Gas Propellants," Ph.D. Dissertation, Aerospace Engineering Department, University of Southampton, Southampton, UK, 2003.

${ }^{14}$ Fearn, D. G., Singfield, A., and Wallace, N. C., "The Operation of Ion Thruster Hollow Cathodes Using Rare Gas Propellants," AIAA/DGLR/JSASS $21^{\text {st }}$ International Electric Propulsion Conference, AIAA Paper 90-2584, 1990.

${ }^{15}$ Gessini, P., Coletti, M., Bevan, N., Intini Marques, R., Michalareas, G., and Gabriel, S. B., "Solar Electric Propulsion for Lunar Transfer", Solicited Paper, $1^{\text {st }}$ Hellenic-European Student Space Science and Technology Symposium, Patras, Greece, 2006.

${ }^{16}$ Grubisic, A., and Gabriel, S. B., "Thrust Characterization of a T5 Hollow Cathode," AIAA/ASME/SAE/ASEE $43^{\text {rd }}$ Joint Propulsion Conference \& Exhibit, AIAA Paper 2007-5194, 2007.

${ }^{17}$ Coletti, M., Grubisic, A., and Wallace, N. C., "European Student Moon Orbiter Solar Electric Propulsion Subsystem Architecture. An All - Electric Spacecraft," 30 International Electric Propulsion Conference, Accepted, 2007. 